Supporting Information for

\title{
The Effect of Substituents at Lewis Acidic Bismuth(III) Centers on its Propensity to Bind a Noble Metal Donor
}

Carolin Tschersich, Santina Hoof, Nicolas Frank, Christian Herwig, Christian Limberg*

Institut für Chemie, Humboldt-Universität zu Berlin, Brook-Taylor-Str. 2, 12489 Berlin, Germany

*corresponding author: christian.limberg@chemie.hu-berlin.de

용 +4930-20937382

$\underline{\text { Table of Contents }}$

$\begin{array}{lr}\text { NMR Spectra } & \text { S 1 }\end{array}$

$\begin{array}{lr}\text { Density Functional Calculations } & \text { S 5 }\end{array}$

$\begin{array}{lr}\text { Crystal Structure Analysis } & \text { S 7 }\end{array}$

$\begin{array}{lr}\text { References } & \text { S 8 }\end{array}$ 


\section{NMR Spectra}
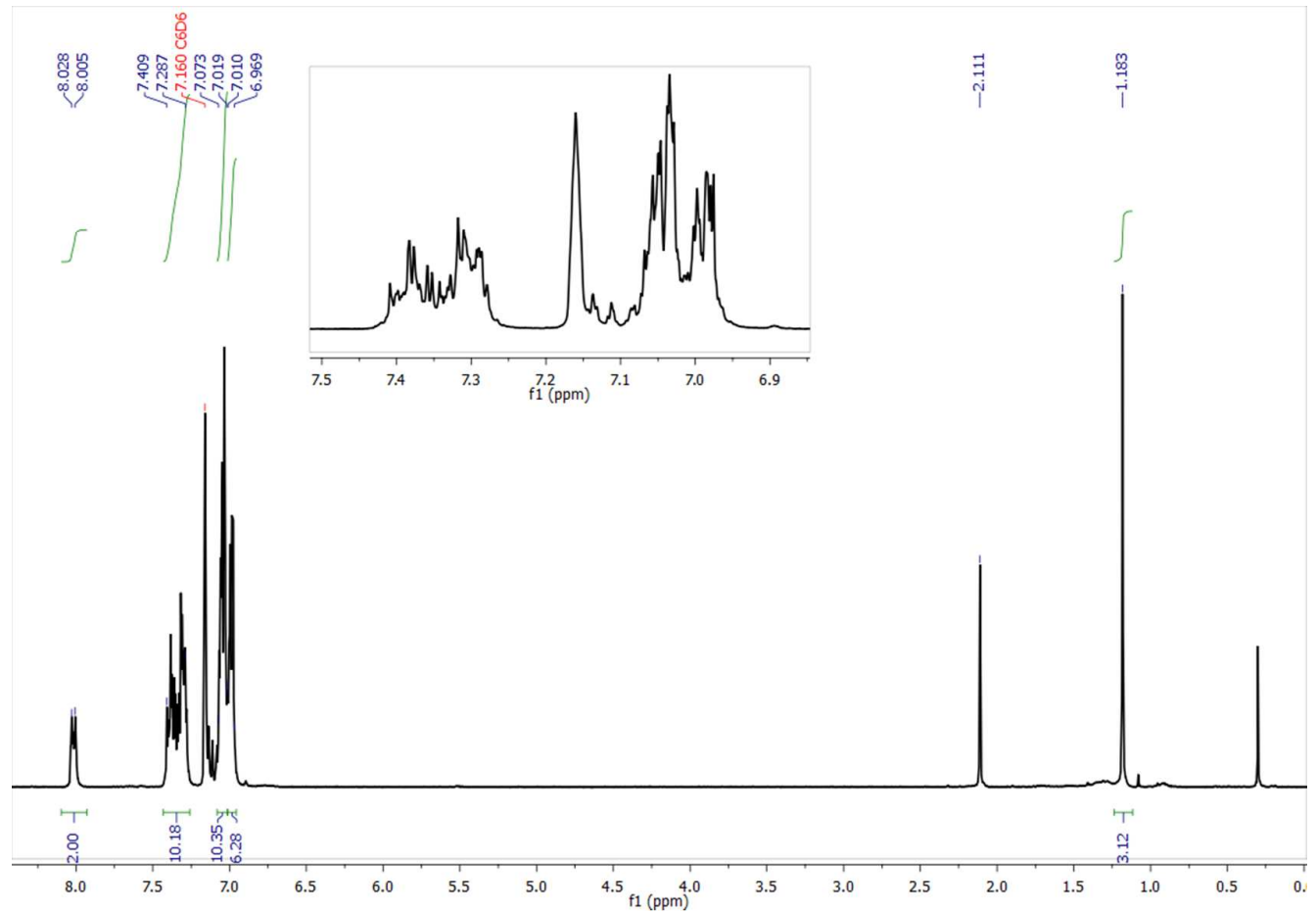

${ }^{1} \mathrm{H}$ NMR spectrum of PBiP-Me in $\mathrm{C}_{6} \mathrm{D}_{6}$.

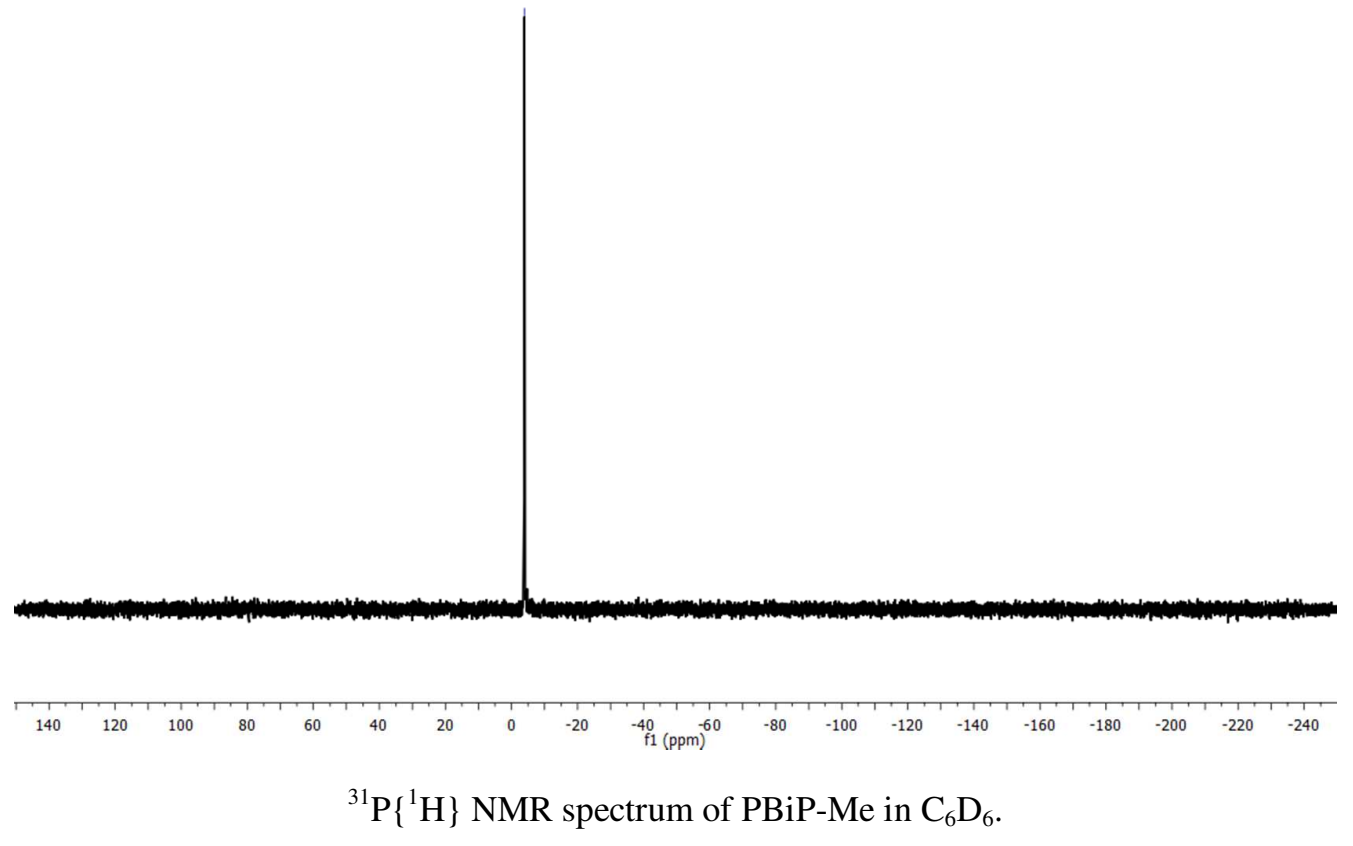




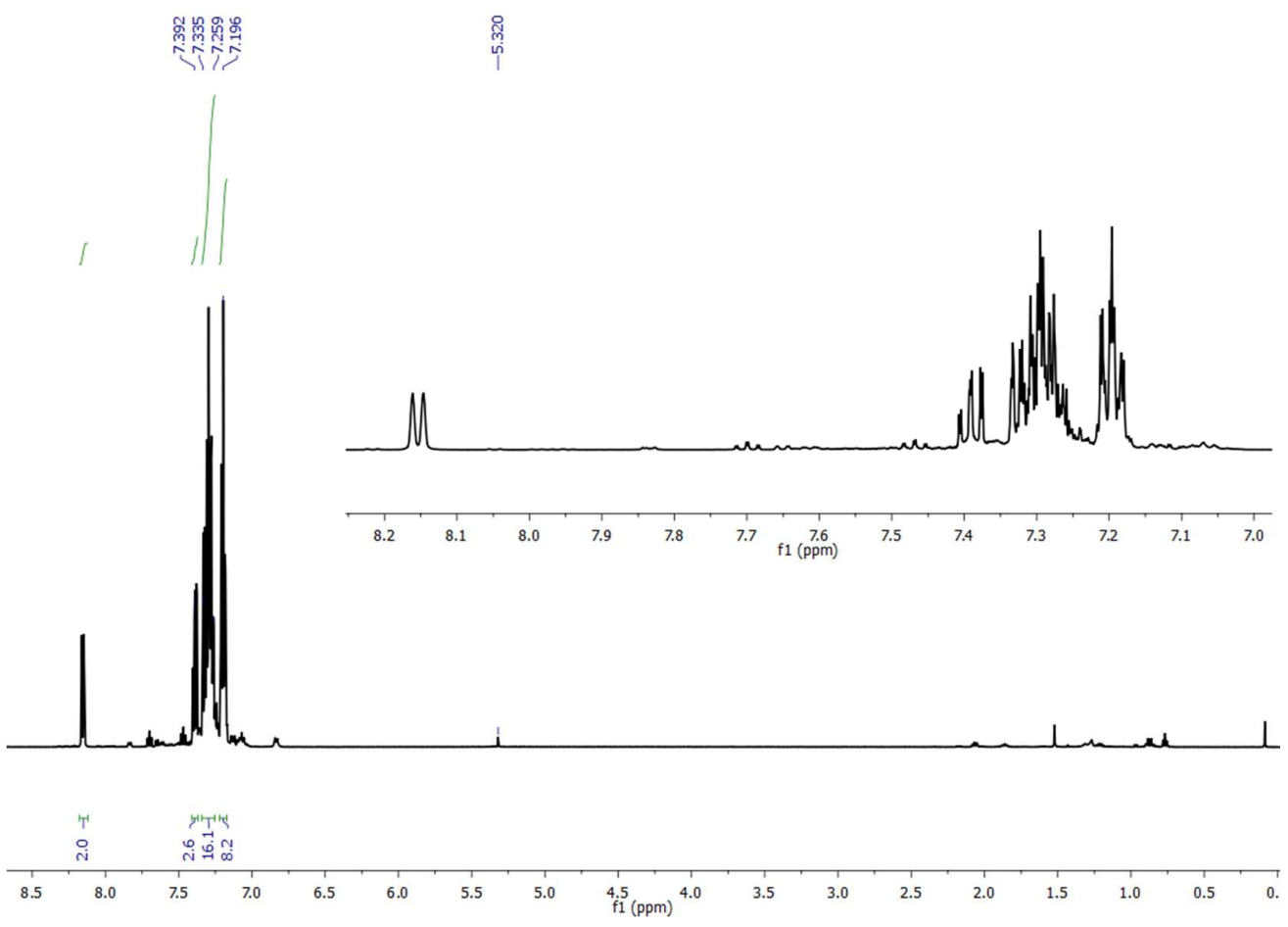

${ }^{1} \mathrm{H}$ NMR spectrum of $\mathrm{PBiP}-\mathrm{C}_{6} \mathrm{~F}_{5}$ in $\mathrm{CD}_{2} \mathrm{Cl}_{2}$.

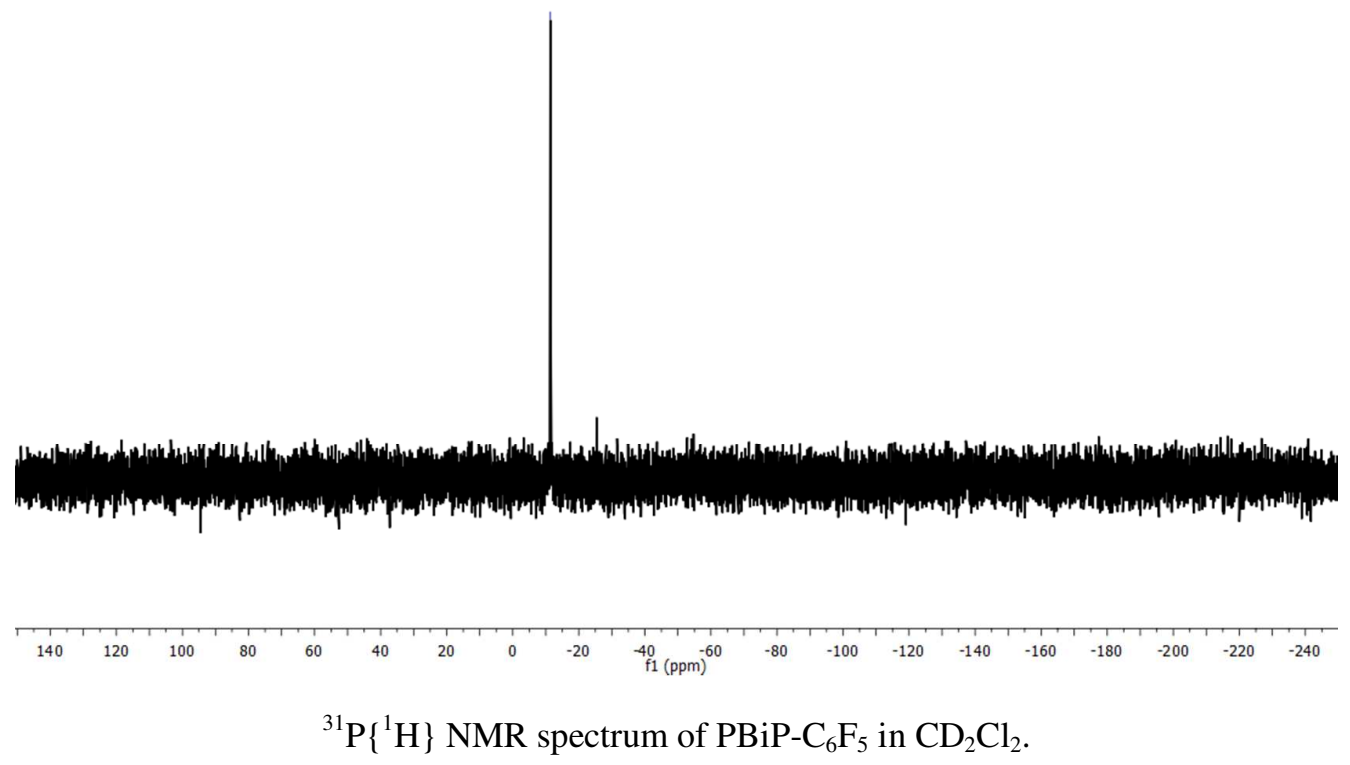




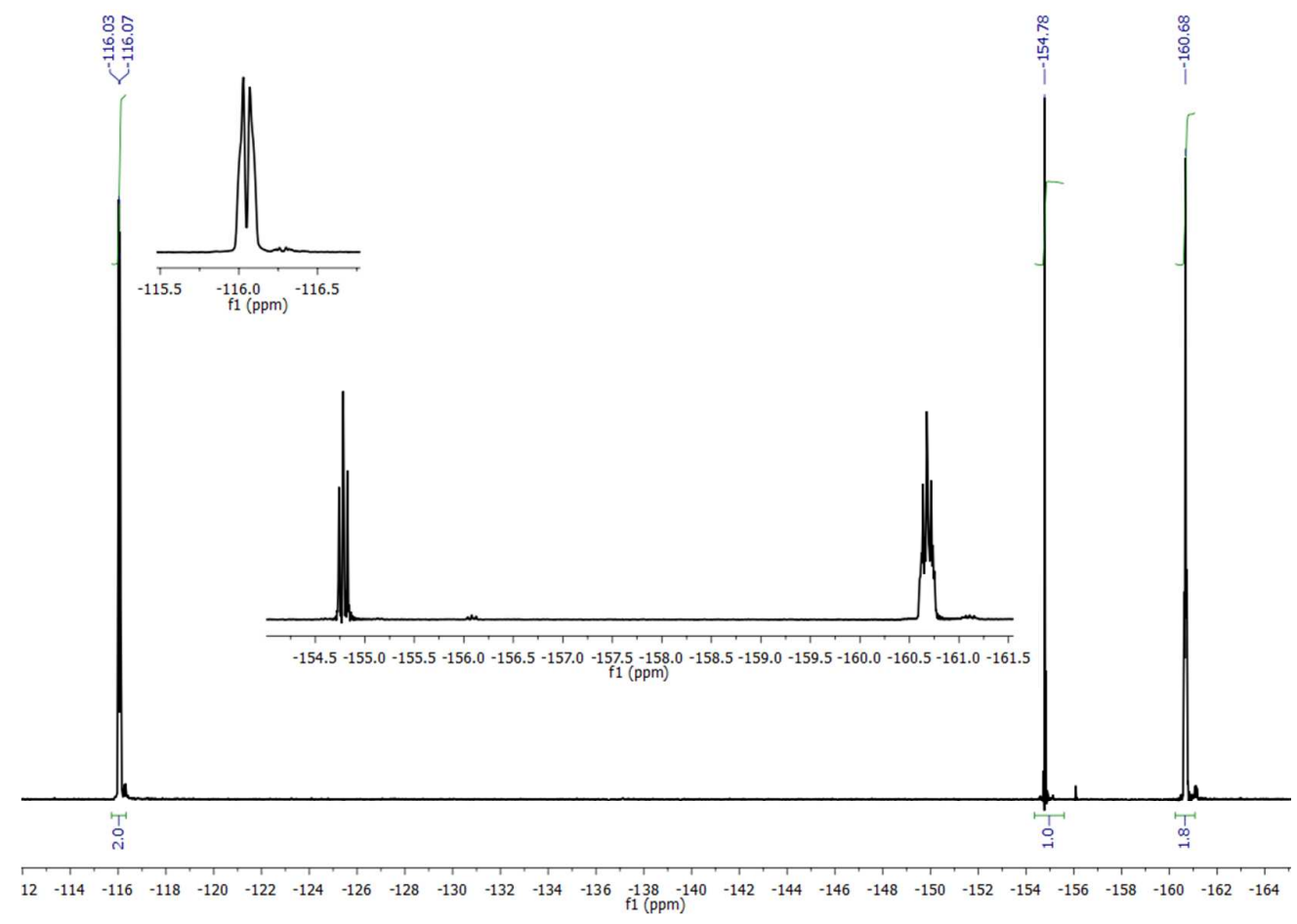

${ }^{19} \mathrm{~F}$ NMR spectrum of $\mathrm{PBiP}-\mathrm{C}_{6} \mathrm{~F}_{5}$ in $\mathrm{CD}_{2} \mathrm{Cl}_{2}$.

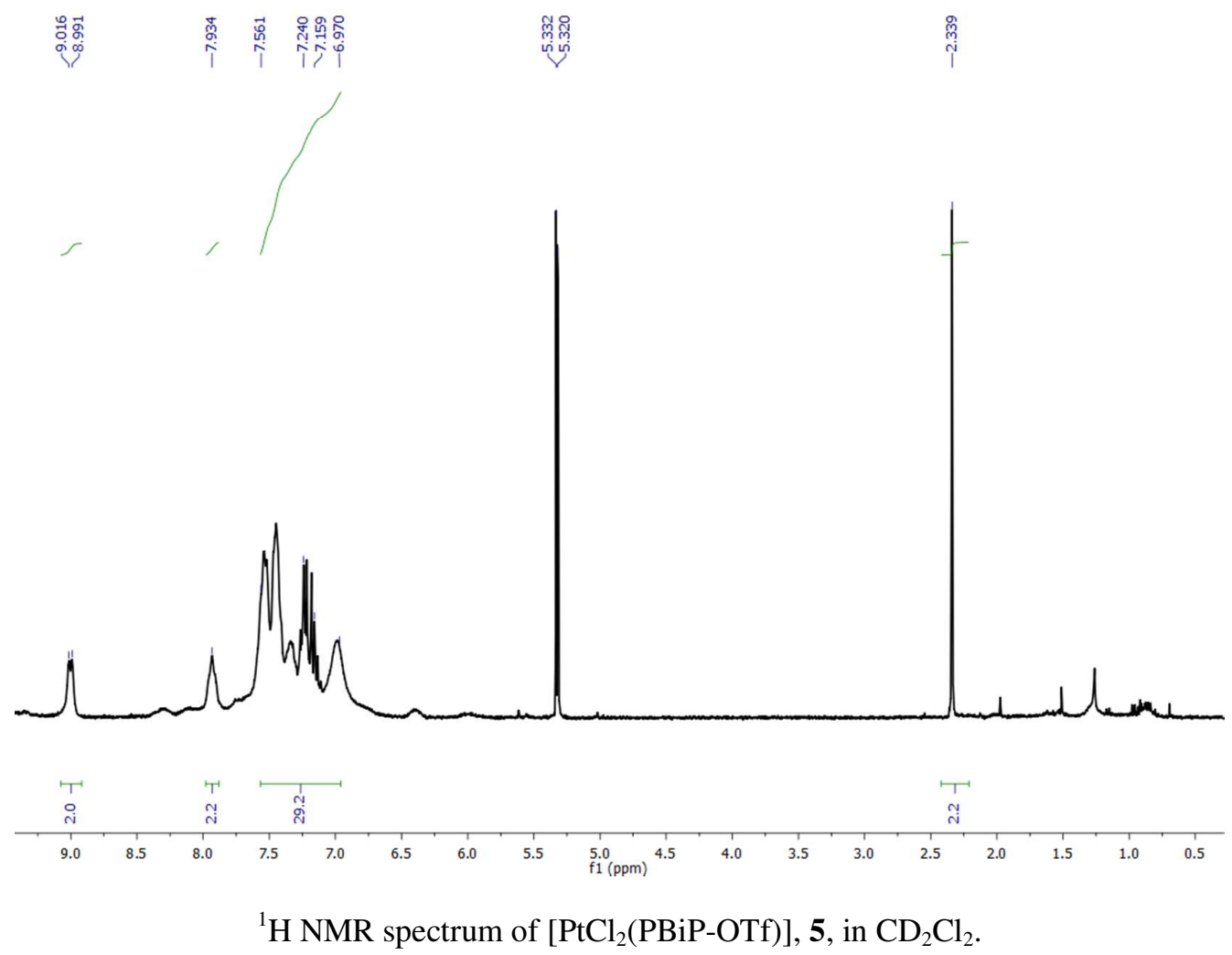




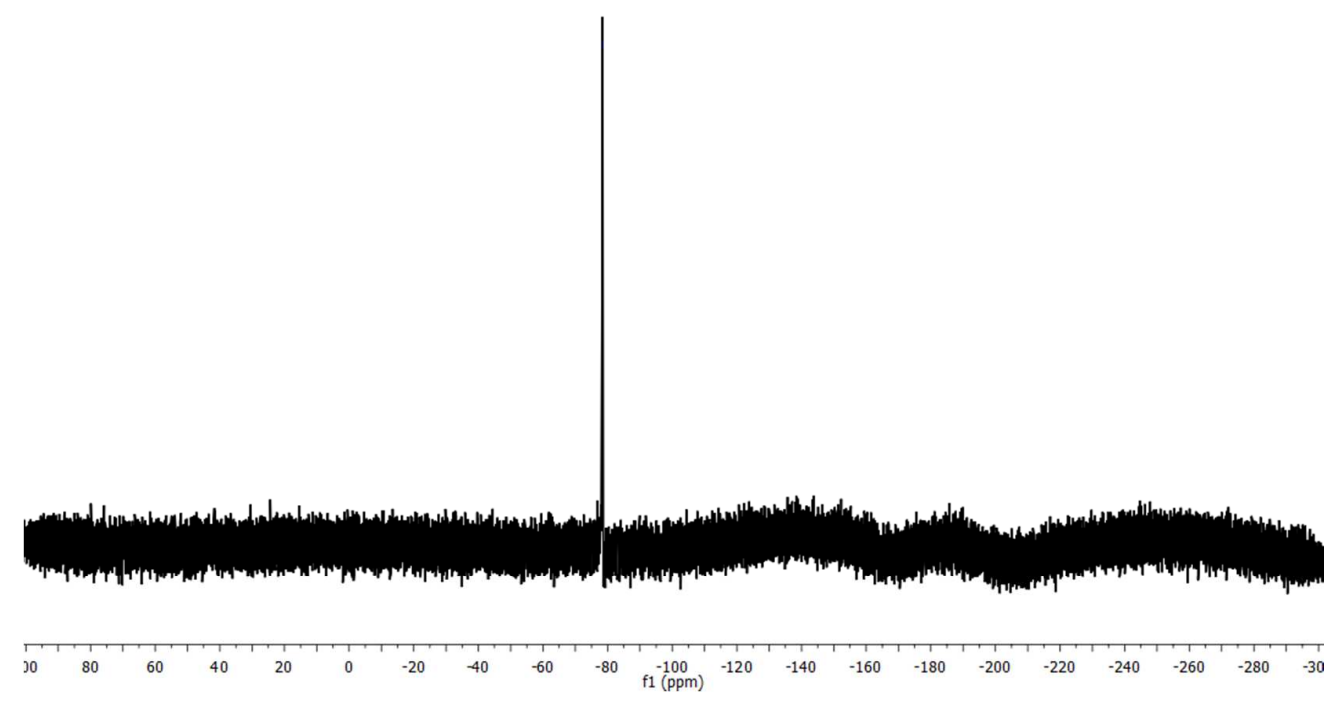

${ }^{19} \mathrm{~F}$ NMR spectrum of $\left[\mathrm{PtCl}_{2}(\mathrm{PBiP}-\mathrm{OTf})\right], \mathbf{5}$, in $\mathrm{CD}_{2} \mathrm{Cl}_{2}$. 


\section{Density Functional Calculations}

Geometry optimizations were performed in redundant internal coordinates without symmetry restrictions using the Gaussian09 program package. ${ }^{[1]}$ The molecular structures of compounds $\left[\mathrm{PtCl}_{2}(\mathrm{PBiP}-\mathrm{X})\right]$ with $3(\mathrm{X}=\mathrm{Me}), \mathbf{4}\left(\mathrm{X}=\mathrm{C}_{6} \mathrm{~F}_{5}\right)$, and $\mathbf{5}(\mathrm{X}=\mathrm{OTf})$ as determined by $\mathrm{X}$-ray diffraction analysis were used as starting points. The B3LYP functional ${ }^{[2]}$ was employed together with the Def2TZVP basis set for all atoms, ${ }^{[3]}$ including Effective Core Potentials for $\mathrm{Bi}$ and $\mathrm{Pt}$ (46 core electrons each), ${ }^{[4]}$ all taken from the EMSL Basis Set Exchange Database. ${ }^{[5]}$ Very tight convergence criteria were chosen for the SCF procedure and a pruned $(99,590)$ "ultrafine" integration grid was used for numerical integrations. Vibrational frequencies for all molecules were computed analytically. The program NBO5. ${ }^{[6]}$ was used for subsequent Natural Bond Orbital (NBO) analysis. Visualization of molecular structures was accomplished with the program Gauss View (Gaussian, Inc.).

For $2(\mathrm{X}=\mathrm{Cl})$ an optimized structure has been published by us before. ${ }^{[7]}$ To ensure comparability, a reoptimization has been done with the currently used version of Gaussian09.

For all molecules the ground state was found to be a singlet state, as expected.

The NBO analysis assigns no Pt-Bi bond, but two strongly polarized covalent $\mathrm{Bi}-\mathrm{C}$ bonds and two strongly polarized Pt-P bonds in each molecule. For the complexes $3(\mathrm{X}=\mathrm{Me})$ and $\mathbf{4}\left(\mathrm{X}=\mathrm{C}_{6} \mathrm{~F}_{5}\right)$ covalent bonds between $\mathrm{Bi}$ and $\mathrm{X}$ (the binding atom of $\mathrm{X}$, respectively) were found, while ionic interactions between $\mathrm{Bi}$ and $\mathrm{X}$ were predicted for $\mathbf{2}(\mathrm{X}=\mathrm{Cl})$ and $\mathbf{5}(\mathrm{X}=\mathrm{OTf})$.

For donor acceptor interactions between occupied $\mathrm{d}(\mathrm{Pt})$ orbitals and empty $6 \mathrm{p}(\mathrm{Bi})$ or $\sigma^{*}(\mathrm{Bi}-\mathrm{C})$ orbitals, NBO deletion energies were calculated by zeroing the Kohn-Sham matrix elements between the corresponding orbital pairs. Pt has 4 occupied $d$ orbitals (the $d_{x 2-y 2}$ orbital is empty). The main interaction comes from the $\mathrm{d}_{\mathrm{z} 2}$ orbital. We have therefore calculated the deletion energies:

A) for the interaction $\mathrm{d}_{\mathrm{z} 2}(\mathrm{Pt}) \rightarrow 6 \mathrm{p}(\mathrm{Bi}) / \sigma^{*}(\mathrm{Bi}-\mathrm{C})$

B) for the interaction of all four occupied d orbitals with the Bi acceptor: $4 x \mathrm{~d}(\mathrm{Pt}) \rightarrow 6 \mathrm{p}(\mathrm{Bi}) / \sigma^{*}(\mathrm{Bi}-\mathrm{C})$

Table S1: Results of density functional calculations for compounds [ $\left.\mathrm{PtCl}_{2}(\mathrm{PBiP}-\mathrm{X})\right]$.

\begin{tabular}{|l|l|l|l|l|}
\hline & $\mathbf{5}(\mathrm{X}=\mathrm{OTf})$ & $\mathbf{2}(\mathrm{X}=\mathrm{Cl})$ & $\mathbf{4}\left(\mathrm{X}=\mathrm{C}_{6} \mathrm{~F}_{5}\right)$ & $\mathbf{3}(\mathrm{X}=\mathrm{Me})$ \\
\hline $\mathrm{d}(\mathrm{Pt}-\mathrm{Bi})$ in $\AA$ (calculated) & 2,9252 & 3,0089 & 3,1115 & 3,1908 \\
\hline $\mathrm{d}(\mathrm{Pt}-\mathrm{Bi})$ in $\AA$ (measured) & 2,7814 & 2,9009 & 3,0521 & 3,062 \\
\hline $\begin{array}{l}\text { Bi-X bond according to } \\
\text { NBO }\end{array}$ & no & no & yes & yes \\
\hline $\begin{array}{l}\text { A) NBO deletion energy } \\
(\mathrm{kcal} / \mathrm{mol})\end{array}$ & $\begin{array}{l}\mathrm{d}_{\mathrm{z} 2}(\mathrm{Pt}) \rightarrow 6 \mathrm{p}(\mathrm{Bi}) \\
73.82\end{array}$ & $\begin{array}{l}\mathrm{d}_{\mathrm{z} 2}(\mathrm{Pt}) \rightarrow 6 \mathrm{p}(\mathrm{Bi}) \\
52.37\end{array}$ & $\begin{array}{l}\mathrm{d}_{\mathrm{z} 2}(\mathrm{Pt}) \rightarrow \sigma^{*}(\mathrm{Bi}-\mathrm{C}) \\
28.65\end{array}$ & $\begin{array}{l}\mathrm{d}_{\mathrm{z} 2}(\mathrm{Pt}) \rightarrow \sigma^{*}(\mathrm{Bi}-\mathrm{C}) \\
16.23\end{array}$ \\
\hline
\end{tabular}




\begin{tabular}{|c|c|c|c|c|}
\hline $\begin{array}{l}\text { B) NBO deletion energy } \\
\text { (kcal/mol) }\end{array}$ & $\begin{array}{l}4 \mathrm{x} \\
\mathrm{d}(\mathrm{Pt}) \rightarrow 6 \mathrm{p}(\mathrm{Bi}) \\
74.23\end{array}$ & $\begin{array}{l}4 x \\
d(P t) \rightarrow 6 p(B i) \\
54.22\end{array}$ & $\begin{array}{l}4 x \\
d(P t) \rightarrow \sigma^{*}(B i-C) \\
33.42\end{array}$ & $\begin{array}{l}4 \mathrm{x} \\
\mathrm{d}(\mathrm{Pt}) \rightarrow \sigma^{*}(\mathrm{Bi}-\mathrm{C}) \\
25.65\end{array}$ \\
\hline
\end{tabular}

The supplemental file CartesianCoordinates.xyz contains the computed Cartesian coordinates of the molecules $3(\mathrm{X}=\mathrm{Me}), \mathbf{4}\left(\mathrm{X}=\mathrm{C}_{6} \mathrm{~F}_{5}\right)$, and $\mathbf{5}(\mathrm{X}=\mathrm{OTf})$ reported in this study. For the Cartesian coordinates of $2(\mathrm{X}=\mathrm{Cl})$ see [7]. The file may be opened as a text file to read the coordinates, or opened directly by a molecular modeling program such as Mercury (version 3.3 or later, http://www.ccdc.cam.ac.uk/pages/Home.aspx) for visualization and analysis. 


\section{Crystal Structure Analysis}

The data collections were performed with a STOE \& CIE (IPDS/2 $\theta$ ) or a BRUKER D8 VENTURE area detector, Mo- $K \alpha$ radiation $(\lambda=0.71073 \AA)$. Multi-scan absorption corrections implemented in PLATON $^{8}$ were applied to the data. The structures were solved by intrinsic phasing method (SHELXT-2013) $)^{9}$ and refined by full-matrix least square procedures based on $F 2$ with all measured reflections (SHELXL-2013) ${ }^{10}$ with anisotropic temperature factors for all non-hydrogen atoms. All hydrogen atoms were added geometrically and refined by using a riding model. CCDC 1437425 (3), 1437426 (4), 1437427 (5), contain the supplementary crystallographic data for this paper. These data can be obtained free of charge from The Cambridge Crystallographic Data Centre via www.ccdc.cam.ac.uk/data_request/cif.

\section{Crystallographic Data for 3. $\mathrm{CH}_{2} \mathrm{Cl}_{2}$ :}

$\mathrm{C}_{37} \mathrm{H}_{31} \mathrm{BiCl}_{2} \mathrm{P}_{2} \mathrm{Pt} \cdot \mathrm{CH}_{2} \mathrm{Cl}_{2}, M_{r}=1097.45$, triclinic, $0.089 \times 0.052 \times 0.019 \mathrm{~mm}, P \overline{1}, a=11.1584(4) \AA, b$ $=11.1612(4) \AA, c=16.6670(7) \AA, \alpha=70.455(2), \beta=77.360(2)^{\circ}, \gamma=68.193(2)^{\circ}, V=1805.51(12) \AA^{3}$, $Z=2, \rho=2.019 \mathrm{~g} \mathrm{~cm}^{-3}, \mu=9.149 \mathrm{~mm}^{-1}$, Mo-K $\mathrm{K}_{\alpha}$ radiation $(\lambda=0.71073 \AA$ ) $, T=100(2) \mathrm{K}, \Theta=2.25-$ $27.17^{\circ}, F_{000}=1040$, reflections collected 30556, reflections unique $7972\left[R_{\text {int }}=0.1180\right], \mathrm{GoF}=1.070$, $R_{1}=0.0475, w R_{2}=0.0757$, largest diff. peak and hole $2.011 /-1.787 \mathrm{e}^{-3}$.

\section{Crystallographic Data for 4 :}

$\mathrm{C}_{42} \mathrm{H}_{28} \mathrm{BiCl}_{2} \mathrm{~F}_{5} \mathrm{P}_{2} \mathrm{Pt}, M_{r}=1164.55$, triclinic, $0.090 \times 0.090 \times 0.080 \mathrm{~mm}, P \overline{1}, a=11.2029(3) \AA, b=$ 11.2650(3) $\AA, c=16.2329(5) \AA, \alpha=86.751(2)^{\circ}, \beta=71.048(2)^{\circ}, \gamma=67.531(2)^{\circ}, V=1785.12(9) \AA^{3}, Z$ $=2, \rho=2.167 \mathrm{~g} \mathrm{~cm}^{-3}, \mu=9.135 \mathrm{~mm}^{-1}$, Mo-K $\alpha$ radiation $(\lambda=0.71073 \AA), T=100(2) \mathrm{K}, \Theta=3.18-$ $26.72^{\circ}, F_{000}=1100$, reflections collected 45472 , reflections unique $7549\left[R_{\text {int }}=0.0742\right]$, GoF $=1.091$, $R_{1}=0.0396, w R_{2}=0.0887$, largest diff. peak and hole $3.350 /-2.141 \mathrm{e}^{-3}$.

\section{Crystallographic Data for $\mathbf{5}$ :}

$\mathrm{C}_{37} \mathrm{H}_{28} \mathrm{BiCl}_{2} \mathrm{~F}_{3} \mathrm{O}_{3} \mathrm{P}_{2} \mathrm{PtS}, M_{r}=1146.56$, monoclinic, $0.015 \times 0.080 \times 0.020 \mathrm{~mm}, P 2{ }_{1} / \mathrm{c}, a=17.8584(4)$ $\AA, b=18.9769(4) \AA, c=10.8121(2) \AA, \beta=90.0619(8)^{\circ}, V=3664.19(13) \AA^{3}, Z=4, \rho=2.078 \mathrm{~g} \mathrm{~cm}^{-3}$, $\mu=8.952 \mathrm{~mm}^{-1}$, Mo-K $\alpha$ radiation $(\lambda=0.71073 \AA), T=100(2) \mathrm{K}, \Theta=2.19-25.40^{\circ}, F_{000}=2168$, reflections collected 43177, reflections unique $6694\left[R_{\text {int }}=0.0753\right], \mathrm{GoF}=1.020, R_{l}=0.0251, w R_{2}=$ 0.0529 , largest diff. peak and hole $1.051 /-1.472 \mathrm{e}^{-3}$. 


\section{References}

(1) Frisch, M. J.; Trucks, G. W.; Schlegel, H. B.; Scuseria, G. E.; Robb, M. A.; Cheeseman, J. R.; Scalmani, G.; Barone, V.; Mennucci, B.; Petersson, G. A.; Nakatsuji, H.; Caricato, M.; Li, X.; Hratchian, H. P.; Izmaylov, A. F.; Bloino, J.; Zheng, G.; Sonnenberg, J. L.; Hada, M.; Ehara, M.; Toyota, K.; Fukuda, R.; Hasegawa, J.; Ishida, M.; Nakajima, T.; Honda, Y.; Kitao, O.; Nakai, H.; Vreven, T.; Montgomery, J. A., Jr.; Peralta, J. E.; Ogliaro, F.; Bearpark, M.; Heyd, J. J.; Brothers, E.; Kudin, K. N.; Staroverov, V. N.; Kobayashi, R.; Normand, J.; Raghavachari, K.; Rendell, A.; Burant, J. C.; Iyengar, S. S.; Tomasi, J.; Cossi, M.; Rega, N.; Millam, J. M.; Klene, M.; Knox, J. E.; Cross, J. B.; Bakken, V.; Adamo, C.; Jaramillo, J.; Gomperts, R.; Stratmann, R. E.; Yazyev, O.; Austin, A. J.; Cammi, R.; Pomelli, C.; Ochterski, J. W.; Martin, R. L.; Morokuma, K.; Zakrzewski, V. G.; Voth, G. A.; Salvador, P.; Dannenberg, J. J.; Dapprich, S.; Daniels, A. D.; Farkas, Ö.; Foresman, J. B.; Ortiz, J. V.; Cioslowski, J.; Fox, D. J. Gaussian 09, Revision C.01; Gaussian, Inc., Wallingford CT, 2010.

(2) a) Becke, A. D. Phys. Rev. A 1988, 38, 30983100; b) Lee, C.; Yang, W.; Parr, R. G. Phys. Rev. B 1988, 37, 785-789; c) Becke, A. D. J. Chem. Phys. 1993, 98, 5648-5652.

(3) Weigend, F.; Ahlrichs, R. Phys. Chem. Chem. Phys. 2005, 7, 3297.

(4) a) Andrae, D.; Haeussermann, U.; Dolg, M.; Stoll, H.; Preuss, H. Theor. Chim. Acta 1990, 77, 123-141; b) Metz, B.; Stoll, H.; Dolg, M. J. Chem. Phys. 2000, 113, 2563-2569.

(5) a) Feller, D. J. Comp. Chem. 1996, 17, 1571-1586; b) Schuchardt, K. L.; Didier, B. T.; Elsethagen, T.; Sun, L.; Gurumoorthi, V.; Chase, J.; Li, J.; Windus, T. L. J. Chem. Inf. Model. 2007, 47, 10451052 .

(6) Glendening, E. D.; Badenhoop, J. K.; Reed, A. E.; Carpenter, J. E.; Bohmann, J. A.; Morales, C. M.; Weinhold, F., Theoretical Chemistry Institute, University of Wisconsin, Madison, WI, 2001; http://www.chem.wisc.edu/ nbo5.

(7) Tschersich, C.; Limberg, C.; Roggan, S.; Herwig, C.; Ernsting, N.; Kovalenko, S.; Mebs, S. Angew. Chem. 2012, 124 (20), 5073-5077.

(8) A. L. Spek, Acta Cryst. 2009, D65, 148-155.

(9) G. M. Sheldrick. SHELXT-2013; University of Göttingen, Germany, 2013.

(10) G. M. Sheldrick. SHELXL-2013; University of Göttingen, Germany, 2013. 